\author{
Bernd Klaeser \\ Michel D. Mueller \\ Ralph A. Schmid \\ Carlos Guevara \\ Thomas Krause \\ Jakub Wiskirchen
}

\section{PET-CT-guided interventions in the management of FDG-positive lesions in patients suffering from solid malignancies: initial experiences}

Received: 18 July 2008

Revised: 16 December 2008

Accepted: 22 December 2008

Published online: 24 February 2009

(C) European Society of Radiology 2009

B. Klaeser · T. Krause $(\bowtie)$.

J. Wiskirchen

Department of Nuclear Medicine, Inselspital, Bern University Hospital and University of Bern,

Bern, Switzerland

e-mail: thomas.krause@insel.ch

Tel.: +41-31-6322656

Fax: +41-31-6323137

\section{D. Mueller}

Department of Obstetrics and

Gynaecology, Inselspital,

Bern University Hospital and

University of Bern,

Bern, Switzerland

\section{R. A. Schmid}

Department of Thoracic Surgery,

Inselspital, Bern University Hospital and University of Bern,

Bern, Switzerland

\section{Guevara}

Institute of Pathology,

University of Bern,

Bern, Switzerland

\author{
J. Wiskirchen \\ Department of Radiology, \\ Neuroradiology, and Nuclear Medicine, \\ University Hospital Tuebingen, \\ Tuebingen, Germany
}

\begin{abstract}
Positron emission tomography-computed tomography (PET-CT) has gained widespread acceptance as a staging investigation in the diagnostic workup of malignant tumours and may be used to visualize metabolic changes before the evolution of morphological changes. To make histology of PET findings without distinctive structural changes available for treatment decisions, we developed a protocol for multimodal image-guided interventions using an integrated PET-CT machine. We report our first experience in 12 patients admitted for staging and restaging of breast cancer, non-small cell lung cancer, cervical cancer, soft tissue sarcoma, and osteosarcoma. Patients were repositioned according to the findings in PET-CT and intervention
\end{abstract}

was planned based on a subsequent single-bed PET-CT acquisition of the region concerned. The needle was introduced under CT guidance in a step-by-step technique and correct needle position in the centre of the FDG avid lesion was assured by repetition of a single-bed PET-CT acquisition before sampling. The metabolically active part of lesions was accurately targeted in all patients and representative samples were obtained in $92 \%$. No major adverse effects occurred. We conclude that PET-CT guidance for interventions is feasible and may be promising to optimize the diagnostic yield of CTguided interventions and to make metabolically active lesions without morphological correlate accessible to percutaneous interventions.

Keywords Image-guided intervention - Percutaneous biopsy · Positron emission tomography PET-CT $\cdot$ FDG

\section{Introduction}

Accurate staging is a prerequisite for an individualised, risk-adapted therapy of cancer patients. In this context, positron emission tomography-computed tomography (PET-CT) has become an inherent part of diagnostic workup, e.g. in staging lung cancer [1], in restaging and patient selection for metastasectomy in colorectal cancer [2] and in staging and restaging of breast cancer [3, 4]. As PET-for example using the glucose analogue $\left[{ }^{18} \mathrm{~F}\right]$ fluorodeoxyglucose (FDG) — visualizes metabolic changes in tumour tissues, even lesions without morphological 
correlates can be depicted. In a clinical context, early and reliable detection of distant metastases is crucial, as these change stage grouping and thus the therapeutic approach from a curative to a palliative intention. As patients with distant metastatic disease usually have incurable disease, the local and systemic therapies tend to be less aggressive and associated with fewer side effects than those used for curative purposes. Therefore, optimal staging with PET-CT may prevent patients in a palliative situation from receiving futile but burdensome therapies at the expense of quality of life.

However, despite the overall good sensitivity and specificity of PET-CT, e.g. $83 \%$ and $91 \%$ for lung cancer staging [5], a number of benign conditions mimicking metastatic FDG uptake have been described, eventually leading to an overstaging and undertreatment of patients [6]. Lardinois et al. found that up to half of solitary extrapulmonary FDG accumulations found during PET-CT staging of lung cancer may represent unrelated malignancies or benign disease, e.g. benign tumours or inflammatory changes [7]. The histological assessment of singular hypermetabolic lesions thus appears to be imperative, especially in the absence of confirmative structural changes. Percutaneous image-guided biopsies are widely used as a minimally invasive alternative to open surgical biopsy. However, lesions which are only visible on PET images may not be accessible to CT-guided biopsy. We therefore developed a combined approach using the metabolic and topographic information of PET together with CT navigation. We report our first experience in performing multimodal image-guided interventions using a protocol with an integrated PET-CT device.

\section{Materials and methods}

\section{Patients}

Interventions under PET-CT guidance in 12 patients ( 7 female, 5 male) were retrospectively reviewed in accordance with the regulations of the local ethics committee. All patients were referred to the Department of Nuclear Medicine for PET-CT staging or restaging of malignancies. Median age of patients was 59 years (mean 57 years, range 18-84 years). Patients suffered from or had a history of breast cancer $(n=5)$, non-small cell lung cancer (NSCLC, $n=4)$, cervical cancer $(n=1)$, pelvic soft tissue sarcoma $(n=1)$ and osteosarcoma of the femur $(n=1)$.

\section{FDG PET-CT}

Positron emission tomography was performed using an integrated PET-CT system which combines a 16-slice helical CT machine with high-resolution PET (Biograph 16 Hi-Rez, Siemens Medical Solutions). All patients fasted for at least $6 \mathrm{~h}$ before examination. Image acquisition for a
PET-CT from neck to pelvis was done 90 min after intravenous injection of 5-7 MBq FDG/ $\mathrm{kg}$ body weight with a low-dose unenhanced computed tomography (reconstructed slice thickness $2 \mathrm{~mm}$ ) and emission images were obtained 3 min per bed position (length of body examined $16.2 \mathrm{~cm})$ from the neck to the pelvis $(5-7$ bed positions). When interventions were performed on the day of PET-CT, no additional FDG was injected, but the acquisition time for emission images $120-180 \mathrm{~min}$ after injection was prolonged to $5 \mathrm{~min}$ per bed position to assure adequate image quality. For interventions on a separate day 3-5 MBq FDG $/ \mathrm{kg}$ body weight was injected and the acquisition time was $5 \mathrm{~min}$ per bed position 60-90 $\mathrm{min}$ after injection. Blood glucose level was below $10 \mathrm{mmol} / \mathrm{l}$ in all patients. Images were evaluated qualitatively and maximum standardized uptake values were measured. No SUV cutoff values for the distinction of benign from malign lesions were applied.

\section{Intervention}

The indication for biopsy was based on findings in FDG PET-CT and the consultation with the treating physician. Only lesions of potential impact on the further therapeutic concept were biopsied. Punctures were performed under supervision of or by an experienced interventional radiologist. All patients gave written consent before the intervention. Patients underwent biopsy either on the same day as whole body PET-CT or were scheduled on a separate day. All patients were repositioned depending on the localization of the lesion concerned and a PET-CT acquisition of a single bed position of the region of interest was obtained in prone or supine position. Based on this PET-CT dataset (length of body examined $16.2 \mathrm{~cm}$ ), the access path was defined based according to the presentation of the metabolically active lesion of interest. Interventions were performed under aseptic conditions, local anaesthesia and additional sedation with midazolam, if necessary. The needle was introduced under CT guidance in a step-by-step technique with respect to individual anatomical landmarks visible in CT, as defined according to the PET-CT dataset acquired in biopsy position. The correct needle position in the centre of the FDG avid lesion was assured by repetition of a single-bed PET-CT acquisition before sampling. If necessary, the biopsy needle was repositioned depending on the result of the control images. Bone and soft tissue biopsy systems were chosen depending on localization and depth of the lesion. One or more samples were obtained. In one patient a soft tissue mass was marked with a localization coil and a localization wire before resection. Single-bed PET-CT acquisition was repeated in small lesions after sampling to evaluate the sampling of the active tissue. Total time for single bed PETCT was $12 \mathrm{~min}$ in nine patients and $18 \mathrm{~min}$ in three patients for two and three repetitive acquisitions, respectively. 


\section{Results}

A total of 14 sites were punctured in 12 patients. PET-CTguided intervention was technically successful, i.e. the metabolically active part of lesions was successfully targeted in all patients and in 12 of 13 metabolically active sites representative tissue samples could be obtained $(92 \%)$. In 10 of 12 patients a definite diagnosis was possible based on the obtained samples. According to CT, the median diameter of all lesions punctured was $9 \mathrm{~mm}$ (mean $16 \mathrm{~mm}$, range $7-40 \mathrm{~mm}$ ). The median diameter of bone lesions was $8 \mathrm{~mm}$ (mean $9 \mathrm{~mm}$, range $8-10 \mathrm{~mm}$ ), while soft tissue lesions had a median diameter of $15 \mathrm{~mm}$ (mean $19 \mathrm{~mm}$, range $7-40 \mathrm{~mm}$ ). Two lesions were not delineable in CT; seven lesions did not present a pathological appearance in $\mathrm{CT}$ and could be delineated retrospectively in CT together with the PET information. Two lesions were centrally necrotic. The mean depth of lesions was $74 \mathrm{~mm}$ (median $70 \mathrm{~mm}$ ) without major differences between bone and soft tissue lesions (Table 1).

\section{Bone biopsies}

Bone biopsies were performed in four patients with breast cancer (two primary staging, two restaging) and in one patient with NSCLC (primary staging) and osteosarcoma (staging), respectively. All patients with breast cancer presented with solitary bone lesions. In two patients lesions had no characteristic morphological appearance for metastatic disease, one had increased FDG uptake in the posterior part of the left acetabulum without any morphological correlate (Fig. 1) and one had minor changes in a vertebral body incongruent with the activity distribution. In patients 2,4 and 5 diagnosis of bone metastases was confirmed histologically. Patient 5 had only a subtle bone marrow infiltration by cells originating from breast cancer. Patient 6 with osteosarcoma of the right femur and a suspicious rib lesion in an initial skeletal scintigram was biopsied after one cycle of chemotherapy according to a tumour board decision despite absence of high FDG uptake in PET-CT. In this case, according to the morphological and metabolic appearance only sclerosis was detected, but no sign of malignancy. However, the result remained inconclusive and taking into account a potentially false-negative biopsy a rib resection was performed 4 months later, showing scattered tumour cells in the specimen. In patient 8 with newly diagnosed NSCLC bilateral symmetric pulmonary, hilar and mediastinal uptake suggested sarcoidosis. PET-CT-guided biopsy revealed a bone metastasis in a morphologically indeterminate lesion scaled $10 \mathrm{~mm}$ in a thoracic vertebral body. In patient 10 , who had concomitant uptake in the right lung and in hilar lymph nodes (differential

Table 1 Patient characteristics

\begin{tabular}{|c|c|c|c|c|c|c|c|c|}
\hline & \multirow[t]{2}{*}{ Diagnosis } & \multirow[t]{2}{*}{ Localization of biopsy } & \multicolumn{3}{|l|}{ Lesion } & \multirow[t]{2}{*}{ Histology } & \multicolumn{2}{|l|}{ Success } \\
\hline & & & Diameter & Depth (cm) & Metabolism & & Technical & Clinical \\
\hline 1 & Cervical cancer & Mediastinum & $2 \mathrm{~cm}$ & 9 & + & Cervical cancer & Yes & Yes \\
\hline 2 & Breast cancer & Lumbar spine & $8 \mathrm{~mm}^{* * *}$ & 11 & + & Breast cancer & Yes & Yes \\
\hline 3 & Soft tissue sarcoma & Lung & $11 \mathrm{~cm}^{*}$ & 7 & + & Soft tissue sarcoma & Yes & Yes \\
\hline 4 & Breast cancer & Scapula & $8 \mathrm{~mm}^{* * *}$ & 3 & + & Breast cancer & Yes & Yes \\
\hline 5 & Breast cancer & Acetabulum & $* *$ & 10 & + & Breast cancer & Yes & Yes \\
\hline 6 & Osteosarcoma & Rib & $8 \mathrm{~mm}$ & 6 & - & Sclerosis & Yes & No \\
\hline 7 & Breast cancer & $\begin{array}{l}\text { Parasternal, clavicular and } \\
\text { paravertebral LN }\end{array}$ & $\begin{array}{l}7 \mathrm{~mm}^{* * *} \\
7 \mathrm{~mm}^{* * *} \\
9 \mathrm{~mm}^{* * *}\end{array}$ & $\begin{array}{l}7 \\
4 \\
6\end{array}$ & + & Breast cancer & Yes & Yes \\
\hline 8 & NSCLC & Thoracal spine & $10 \mathrm{~mm}^{* * *}$ & 7 & + & NSCLC & Yes & Yes \\
\hline 9 & NSCLC & Thorax & $5 \times 3 \mathrm{~cm}$ & 3 & + & NSCLC & Yes & Yes \\
\hline 10 & Breast cancer & Thoracal spine & $* *$ & 8 & + & Breast cancer & Yes & No \\
\hline 11 & NSCLC & Supraclavicular LN & $7 \mathrm{~mm} * * *$ & 5 & + & Sinus histiocytosis & Yes & Yes \\
\hline 12 & NSCLC & Pelvic LN & $4 \mathrm{~cm}$ & 13 & + & Prostate Cancer & Yes & Yes \\
\hline
\end{tabular}

*Subtotal necrosis, diameter of the metabolically active region approx. $2 \times 3 \mathrm{~cm}$

**Not delineable in CT

$* * *$ No distinctive morphological alterations in $\mathrm{CT}$ 
diagnosis lung cancer), atypical cells were found, but a definite diagnosis was not possible due to absence of any marker expression in immunohistochemical staining after decalcification of the specimen. Subsequent lung resection showed the same cell type again, but diagnosis of breast cancer recurrence was finally possible.

\section{Soft tissue biopsies}

Soft tissue interventions included three patients with suspected or proven NSCLC (primary staging) and one patient with cervical cancer (restaging), breast cancer (restaging) and a pararectal soft tissue sarcoma (restaging), respectively. In patient 1 with a history of cervical cancer and in patient 7 with breast cancer second malignancies were taken into account based on the distribution and pattern of FDG uptake in PETCT. However, recurrences of the known primary tumours were confirmed in both patients. Patient 3 with a history of a pararectal soft tissue sarcoma presented with a total atelectasis of his left lung and pleural effusion. A preceding CT was equivocal and an infectious cause of the basal tumour mass had been suggested. PET-CT clearly detected areas with increased FDG uptake, i.e. vital tissue in the collapsed lung, which were biopsied and showed poorly differentiated sarcomatous tissue (Fig. 2). In three further patients with NSCLC indications were verification of a chest wall infiltration (patient 9), clarification of FDG uptake in a solitary supraclavicular lymph node (patient 11, sinus histiocytosis) and in a singular pelvic lymph node (patient 12, prostate cancer).

\section{Side effects and complications}

Seven of 12 patients were treated on an outpatient basis and were released after 2-4 h of supervision; five patients were hospitalized overnight for logistic reasons. All patients were released in good physical status. No serious adverse effects (e.g. major bleeding, pneumothorax, infection) occurred. In two patients venous bleeding developed which stopped spontaneously in one and was successfully treated in another patient on acetylsalicylic acid at the time of biopsy by injecting a blood clot through the puncture cannula. An unenhanced CT image revealed a small cshaped hematoma around the lymph node which had been punctured. Among four patients with chest interventions no pneumothorax occurred. Interventions were well tolerated under local anaesthesia. Four patients had midazolam 2$5 \mathrm{mg}$ for short-term sedation.

\section{Discussion}

Positron emission tomography has gained widespread acceptance as a staging investigation in the diagnostic workup of many tumours and was shown to have the potential to clarify true stage grouping in up to one third of patients after conventional staging $[1-4,8-10]$. However, false-positive results may occur in about $10 \%$ of all patients [11-13]. Therefore, having the therapeutic impact of distant metastases in mind, suspicious PET-CT findings have to be evaluated prudently. Assumed metastases may only be regarded as relevant for therapeutic decisions if they can be verified histologically. This is especially true in cases of singular and morphologically indeterminate findings exclusively visualized by PET.

Image-guided punctures are well established in the radiological community. Even though success rates for CTguided biopsies of morphologically clearly circumscribed lesions are reported to range between 70 and $90 \%[14,15]$, the false-negative rate of only PET positive lesions may be substantially higher. As a result of the lack of functional information $\mathrm{CT}$ puncture can be negative even though a
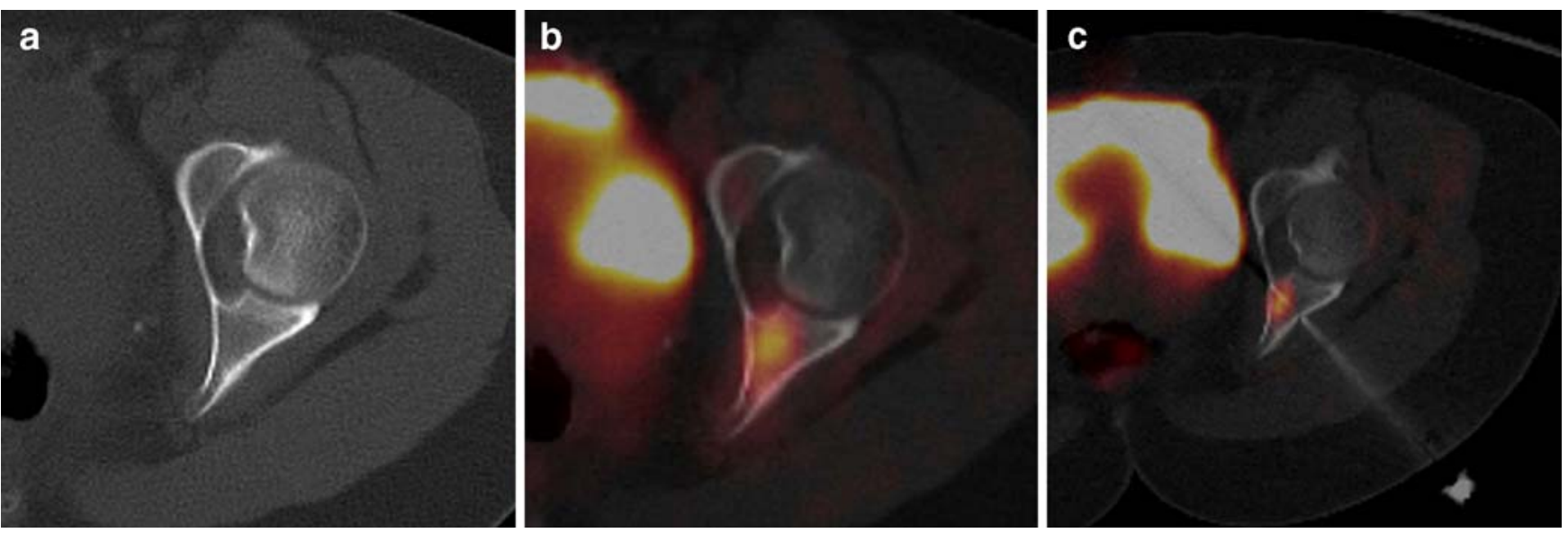

Fig. 1 PET-CT-guided biopsy of a bone lesion not visible in CT. The semiquantitatively measured activity (SUV) in the region concerned is reduced after biopsy. Histology revealed a breast cancer metastasis. a CT, b PET-CT, c PET-CT with decreased activity of the lesion after biopsy 
Fig. 2 Determination of optimal location for sampling, i.e. vital tumour tissue, in a subtotal necrotic pulmonary mass according to metabolic activity. Histology revealed a metastasis of a soft tissue sarcoma. a CT, $\mathbf{b}$ PET-CT-guided biopsy of the focus with highest FDG uptake
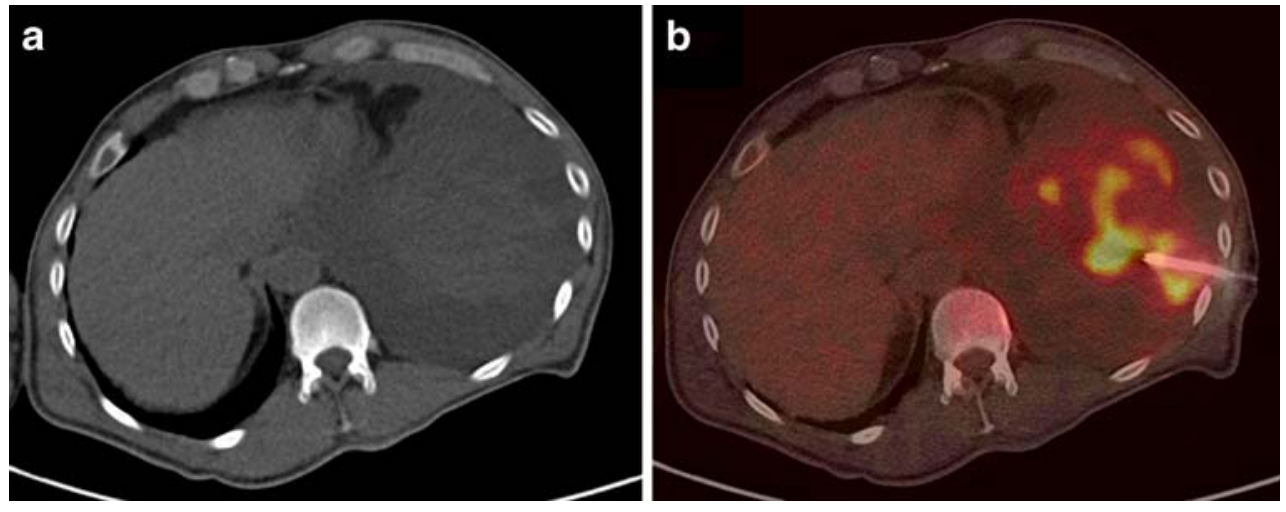

malignant disease is present, e.g. when puncturing the wrong nodule or when taking a biopsy from a part of a lesion which is not representative. Problems may occur when PET imaging and interventions are performed by different persons, at different time points, using different devices and having the patient positioned differently. In order to increase the success rate of image-guided biopsies and to decrease the number of second or even third imageguided biopsies, it would be helpful to puncture the part of the lesion presenting the strongest metabolism.

We describe our first experience with a protocol for PETCT-guided interventions. We used an integrated PET-CT system and combined two well-established methods-PET imaging and CT-guided navigation - in close collaboration with an interdisciplinary team of specialists in nuclear medicine and interventional radiology to make lesions, which are characterized by metabolic properties more than by morphological abnormalities, accessible for histological verification.

To the best of our knowledge this is the first report describing PET-CT-guided interventions employing integrated PET-CT in patients suffering from solid tumours. In our series of 12 consecutive patients PET-CT-guided intervention was technically successful in all patients. Needle positioning guided by PET-CT, similar to what has been described by Veit et al. in an ex vivo liver model [16], proved to be feasible and no relevant complication occurred in a clinical routine setting. PET-CT-guided interventions had an additional clinical impact in $50 \%$ of the patients.

The advantages of this new approach are obvious. PETCT-guided biopsies offer the possibility to directly puncture the metabolically active tissue of relevance-even if structural landmarks are missing. Beside this, the metabolically active, i.e. vital, part of a lesion can be targeted to make sure that representative samples are obtained and futile biopsy attempts, and the inherent strain for the patient, may be avoided. According to our experience, even small lesions without any morphologically distinctive feature can be punctured reliably and the correct needle positioning can be ascertained by a repeated PET-CT acquisition of the region concerned before tissue sampling.

A certain drawback of PET-CT-guided biopsy in a stepby-step technique is the fact that interventions are rather time consuming and, given the occupancy of the PET-CT system, cost-intensive. Although we achieved a high diagnostic accuracy in our initial 12 patients without any major side effects, we are aware that our retrospective evaluation bears several limitations. First of all, the sample size is relatively small. In a larger series, the rate of complications may be expected in the range of CT-guided punctures, as methodology of PET-CT-guided interventions is similar to the established CT-guided punctures. Potentially, the prolonged time needed and inconvenience for the patient might even lead to an increased number of side effects, although this was not observed in our small series.

\section{Conclusion}

PET-CT guidance is feasible and may be a promising new technique in patients with otherwise not or at least not reliably accessible metabolically active lesions. PET-CTguided interventions may help to reduce the risk of possible under- or overtreatment resulting from false stage grouping even in borderline cases of metabolically active lesions without morphological correlate. Further evaluation of clinical impact and cost-efficiency in larger trials is necessary.

Acknowledgements B.K., T.K. and J.W. were supported by a local quality assurance project. 


\section{References}

1. Lardinois D, Weder W, Hany TF et al (2003) Staging of non-small-cell lung cancer with integrated positron-emission tomography and computed tomography. N Engl J Med 348:2500-2507

2. Herbertson RA, Lee ST, Tebbutt N, Scott AM (2007) The expanding role of PET technology in the management of patients with colorectal cancer. Ann Oncol 18:1774-1781

3. Klaeser B, Wiederkehr O, Koeberle D et al (2007) Therapeutic impact of 2[fluorine-18]fluoro-2-deoxy-D-glucose positron emission tomography in the pre- and postoperative staging of patients with clinically intermediate or high-risk breast cancer. Ann Oncol 18:1329-1334

4. Piperkova E, Raphael B, Altinyay ME et al (2007) Impact of PET/CT in comparison with same dame contrast enhanced CT in breast cancer management. Clin Nucl Med 32:429-434

5. Margolis DJ, Hoffman JM, Herfkens RJ et al (2007) Molecular imaging techniques in body imaging. Radiology 245:333-356
6. Metser U, Even-Sapir E (2007) Increased (18)F-fluorodeoxyglucose uptake in benign, nonphysiologic lesions found on whole-body positron emission tomography/computed tomography (PET/CT): accumulated data from four years of experience with PET/CT. Semin Nucl Med 37:206-222

7. Lardinois D, Weder W, Roudas $\mathrm{M}$ et al (2005) Etiology of solitary extrapulmonary positron emission tomography and computed tomography findings in patients with lung cancer. J Clin Oncol 23:6846-6853

8. Loft A, Berthelsen AK, Roed H et al (2007) The diagnostic value of PET/CT scanning in patients with cervical cancer: a prospective study. Gynecol Oncol 106:29-34

9. Kitajima K, Murakami K, Yamasaki E et al (2008) Performance of integrated FDG-PET/contrast-enhanced CT in the diagnosis of recurrent ovarian cancer: comparison with integrated FDG-PET/ non-contrast-enhanced CT and enhanced CT. Eur J Nucl Med Mol Imaging 35:1912-1920

10. Völker T, Denecke T, Steffen I et al (2007) Positron emission tomography for staging of pediatric sarcoma patients: results of a prospective multicenter trial. J Clin Oncol 25:5435-5441

11. Antoch G, Saoudi N, Kuehl H et al (2004) Accuracy of whole-body dual-modality fluorine-18-2-fluoro-2deoxy-D-glucose positron emission tomography and computed tomography (FDG-PET/CT) for tumor staging in solid tumors: comparison with CT and PET. J Clin Oncol 22:4357-4368
12. Shin DS, Shon OJ, Byun SJ et al (2008) Differentiation between malignant and benign pathologic fractures with F-18fluoro-2-deoxy-D-glucose positron emission tomography/computed tomography. Skeletal Radiol 37:415-421

13. Mahner S, Schirrmacher S, Brenner W et al (2008) Comparison between positron emission tomography using 2[fluorine-18]fluoro-2-deoxy-D-glucose, conventional imaging and computed tomography for staging of breast cancer. Ann Oncol 19:1249-1254

14. Huch K, Röderer G, Ulmar B, Reichel H (2007) CT-guided interventions in orthopedics. Arch Orthop Trauma Surg 127:677-683

15. Lis E, Bilsky MH, Pisinski L et al (2004) Percutaneous CT-guided biopsy of osseous lesion of the spine in patients with known or suspected malignancy. AJNR 25:158-188

16. Veit P, Kuehle C, Beyer T et al (2006) Accuracy of combined PET/CT in image-guided interventions of liver lesions: an ex-vivo-study. World J Gastroenterol 12:2388-2393 